\title{
An improved and highly efficient geometry for facemasks
}

Christopher Cappa ( $\nabla$ cdcappa@ucdavis.edu )

University of California, Davis https://orcid.org/0000-0002-3528-3368

\section{William Ristenpart}

University of California, Davis

\section{Santiago Barreda}

University of California, Davis

\section{Nicole Bouvier}

Icahn School of Medicine

\section{Anthony Wexler}

University of California, Davis https://orcid.org/0000-0003-1565-814X

\section{Sanziana Roman}

University of California, San Francisco

The San Francisco Opera Costume Department

The San Francisco Opera

\section{Letter}

Keywords: face masks, face coverings, respiratory disease

Posted Date: March 15th, 2021

DOl: https://doi.org/10.21203/rs.3.rs-322107/v1

License: (1) (1) This work is licensed under a Creative Commons Attribution 4.0 International License.

Read Full License 


\section{Title: An improved and highly efficient geometry for facemasks}

Authors: Christopher D. Cappa ${ }^{1, *}$, The San Francisco Opera Costume Department ${ }^{2, \dagger}$, William D. Ristenpart, ${ }^{3}$ Santiago Barreda ${ }^{4}$ Nicole M. Bouvier, ${ }^{5}$ Anthony S. Wexler, ${ }^{6}$ Sanziana A. Roman ${ }^{7, *}$

Affiliations:

${ }^{1}$ Dept. of Civil and Environmental Engineering, Univ. of California Davis; 1 Shields Ave., Davis, CA 95616 USA

${ }^{2}$ Costume Department, San Francisco Opera; 301 Van Ness Ave, San Francisco, CA 94102, USA

${ }^{3}$ Dept. of Chemical Engineering, Univ. of California Davis; 1 Shields Ave., Davis, CA 95616 USA.

${ }^{4}$ Dept. of Linguistics, Univ. of California Davis; 1 Shields Ave., Davis, CA 95616 USA.

${ }^{5}$ Department of Medicine, Division of Infectious Diseases, Icahn School of Medicine at Mount Sinai, 1 Gustave

L. Levy Place, New York, NY 10029, USA

${ }^{6}$ Dept. of Mechanical Engineering, Univ. of California Davis; 1 Shields Ave., Davis, CA 95616 USA.

${ }^{7}$ Department of Surgery, Univ. of California, San Francisco; 1660 Divisadero St., San Francisco, CA 94143 USA

$\dagger$ Includes: Amy Ashton-Keller, Daniele McCartan, Galen Till, Jai Alltizer

*Corresponding authors: cdcappa@ucdavis.edu or Sanziana.Roman@ucsf.edu

\section{Abstract}

Wearing masks and face coverings helps reduce transmission of respiratory diseases. Various mask designs exist that target different populations, occupations, and environments. Here, we present evidence that a new facemask design, originally made for professional singers, offers advantages that will strongly benefit the public. Specifically, we show that with proper fitting the reusable cloth singing mask achieves overall filtering efficiencies for both exhaled expiratory aerosols and for inhaled ambient aerosols of $>93 \%$, better than surgical masks and approaching N95 respirators. We attribute the high efficiency largely to the mask design, rather than material. The mask design also provides for less inhibited mouth and jaw movement and overall lower humidity within the mask, suggesting it may provide a more palatable, high-efficiency alternative to medical-grade masks for the public.

\section{Main}

Respirators, face masks, and coverings play important roles in protecting wearers in various occupational settings and are important public health tools for reducing transmission of respiratory disease $(1,2)$. Masks differ in style, features, materials, and fit-including their overall level of protection - to account for different exposure conditions and wearer requirements. Often the most high-efficiency masks, such as N95 respirators, are those in the least supply, especially in times of critical need such as during the COVID-19 pandemic (3). When particularly transmissible variants 
of respiratory diseases arise, as is currently the case with the SARS-CoV-2 virus,(4) the need grows

36 for masks for the public having high efficiency, but that have distinct supply chains from medical-

37 grade masks and respirators.

38 Willingness to wear facemasks depends on real and perceived psychological and physiological 39 drawbacks of mask wearing, which includes increased skin temperature, elevated humidity, 40 difficulty breathing, and discomfort when speaking $(5,6)$. New mask designs that help to mitigate 41 these drawbacks may precipitate greater willingness of people to wear masks, especially if such 42 masks have high overall filtration efficiencies. Not all occupations benefit equally from current 43 mask designs owing to the particular nature of their work. One community notably underserved 44 by standard masks is that of performers, singers. Singing and theater performing require mask 45 designs that generally allow for much more jaw movement compared to other professions as well 46 as increased space between the face and the mask material. Thus, any mask appropriate for singing 47 will also be more comfortable for speech, generally. Finally, group singing presents a particular 48 challenge for community transmission of respiratory disease owing to multiple people vocalizing 49 consistently and at the same time (7) (compared to speaking, where people typically take turns) 50 and the loudness of singing compared to normal speech, as the production of potentially virus51 laden aerosols increases with volume $(8,9)$. As such, masks used in conjunction with singing 52 necessarily require relatively high efficiency.

53 Here, we characterize the aerosol-reduction efficacy of a mask designed originally for singing, 54 but that we find has general benefits for wearing by the public. Developed by a surgeon who is a 55 former opera singer (co-author S. A. R.) in partnership with the San Francisco Opera, the singing mask here (Figure 1a,b; see Methods) uniquely allows for facile jaw movement yet maintains overall high efficiency towards emission of expiratory aerosols, even after accounting for leakage 58 flows. Some other masks targeting singers have been introduced during the COVID-19 pandemic. 59 However, while the singing mask here shares some similarities it differs from these others in terms 60 of fit, material, adjustability, and comfort. Importantly, we explicitly characterize the mask 61 efficacy, which is the key factor. In addition, the mask allows the wearer to drink through a straw 62 if needed without removing the mask, which lowers the risk of exposure over removing the mask 63 and could provide benefits for rehearsals or in other situations where drinking occurs (e.g., schools, 64 cafes, bars). The mask also efficiently filters ambient particles, demonstrating substantial 65 protection to the infection-naive wearer as well. Although designed to accommodate singers, the 
high overall mask efficiency (>93\%) suggests that the singing mask can provide a useful

67 alternative to existing high-efficiency masks (e.g., N95's) for the public.

Following experimental methods desscribed in previous studies $(9,10,11$; see Supplemental Material), and consistent with previous findings (8), the observed particle emission rates for singing $\left(\left\langle\dot{N}_{p, \text { sing }}\right\rangle=11.9 \mathrm{p} / \mathrm{s}\right)$ exceeded those for talking $\left(\left\langle\dot{N}_{p, \text { talk }}\right\rangle=3.3 \mathrm{p} / \mathrm{s}\right.$ by about a factor of three when no mask was used (Figure S1). The particle size distributions generally resembled each other for speaking and singing, although singing led to a slight enhancement in the number of particles between about $1 \mu \mathrm{m}$ and $5 \mu \mathrm{m}$ (Figure S2). Singing and talking differ somewhat in their physiological underpinnings. Singing and 'projecting' the voice typically involve a more rapid closing phase of the vocal folds, resulting in more high-frequency energy in the voice-source spectrum and a louder output sound (12). The higher vocal fold velocities, and the higher degree of vocal fold tension required to produce these higher velocities, could both influence the particle sizes and numbers produced by singers. Singing also requires different valving strategies at the larynx to keep subglottal pressure (and loudness) constant throughout a single 'phrase,' which could also result in different particle size distributions between speaking and singing (13). Regardless of the physiological origin, this small variability in the inherent particle size distribution between singing and speaking will have little influence on the efficiency with which the mask reduces emission of particles from expiration to the surrounding environment.

Representative aerosol emission data from one individual singing Ludwig van Beethoven's Ode to Joy with no mask or while wearing various facemasks, including the singing mask, demonstrate that the efficacy of expiratory particle filtration for airflow passing through the mask material varies with mask type (Figure 1c; Figure S3). Notably, the through-mask efficiency for the singing mask is as high as for an N95 and higher than that for two different types of surgical masks, as well as for various cloth masks including two made of the same cloth as the singing mask (see Supplemental Materials). Considering multiple participants, the through-mask reduction efficacy for expiratory aerosols while speaking the Rainbow Passage (14), singing Ode to Joy, and singing other user-selected songs was very high for the singing mask, with $>99.5 \%$ average reduction observed for all activities and the lowest efficiency for a single individual of only $96.5 \%$ (Figure 2a). This high efficiency exceeds that observed for medical-grade surgical masks (Figure 2b) and for KN95 masks during speaking maneuvers (10) but is similar to that for 
an N95 as noted above (Figure 1c). With respect to sound, the mask effectively acts as a low-pass

97 filter (Figure S4), consistent with previous findings on speech and mask wearing (15). The good aerosol filtering differs notably from a homemade cloth masks made from t-shirts - these had

99 particle emission rates higher than observed without the mask owing to shedding of mask fibers,

100 which may act as aerosolized fomites (10). The high filtering efficiency of the singing mask occurs

101 despite considerable shedding when participants intentionally rubbed the mask against itself, (such

102 as might occur with opening/closing the bottom for potential water sipping), with a shedding rate

103 greater than for the homemade cloth masks (10) (geometric mean $=20 \mathrm{p} / \mathrm{s}$ for the singing mask

104 vs. 1 p/s for the homemade mask; Figure S5). Most likely, this distinct lack of shedding during use

105 for the singing mask results from separation of the mask material and the wearers face, thus

106 eliminating contact abrasion of the mask material.

107 Additionally, the mask-face separation also likely contributes to the filtering ability for air that 108 passes through the mask (Figure 1b). By creating distance between the mouth and nose and the 109 mask material, the airstream will expand (16) and interact with a greater area of the mask while 110 decreasing the air velocity (17), which will increase filtering capacity (Figure S6). For example, a 111 single-fiber filtering efficiency of $90 \%$ at $0.5 \mathrm{~cm}$ separation will increase to over $99 \%$ at the singing 112 mask separation of $6 \mathrm{~cm}$ assuming jet expansion. We tested this sampling from one participant 113 while singing Ode to Joy who wore a two-layer and three-layer mask made of the same cloth but 114 having a pleated surgical-mask style, as well as wearing a variation of the singing mask that 115 eliminates the opening for drinking (see Methods; Figure S7). The pleated masks reduced the 116 through-mask particle emission by a substantial, but comparably small, factor of $\sim 14$ compared to $117 \mathrm{a}>500$ fold reduction for the singing masks (Figure 1b). This provides evidence of the key role 118 that the separation of the mask material from the face plays in reducing through-mask expiratory 119 particle emission.

120 The overall mask performance additionally depends on the extent to which particles escape 121 from the edges of the mask; the above discussion only addresses air that passes through the mask 122 material. Any leakage that results from imperfect sealing between the mask and wearers' face 123 reduces all overall mask efficiencies $(11,18,19)$. For high-quality filtering materials, the extent of 124 leakage is the primary determinant of mask efficiency (19). Following from our previous study on 125 surgical mask leakage during speaking (11), we determined the emission rates of particles from 126 the top, bottom, and side quadrants of the singing mask while participants sang Ode to Joy (Figure 
127 S3). The observed particle emission rates from these quadrants exceeded those for the forward, 128 through-the-mask material direction (Figure 3a). Nonetheless, wearing a mask substantially 129 decreases the observed particle emission rates from the no-mask condition in all directions.

130 The greatest between-participant variability in the absolute emission rate occurs for sampling 131 from the mask top quadrant, near the nose (geometric standard deviation $=0.85$ ). This suggests a 132 greater sensitivity of emissions in this direction to how well the mask seals around the nose, which 133 can result from individuals wearing the masks with different tightness. We confirmed this by 134 having one participant sing Ode to Joy while securing the mask with (i) only the ear loops snug, 135 but not tight, (ii) only the ear loops tight, and (iii) the ear loops tight plus the two head straps 136 (Figure S8). With the snug ear loops-only, the observed particle emission rate from the mask top 137 decreased by only a factor of three over no mask. However, with the tight ear loops-only the 138 particle emission rate from the top decreased by a factor of 38 over no mask and with the tight ear 139 loops and the head straps by a factor of 80 over no mask.

140 Sampling from the bottom quadrant yielded the largest with-mask particle emission rates. 141 While this could indicate the greatest leakage flow in this quadrant, the associated particle size 142 distribution differs notably from those observed in the other quadrants (Figure S9). In particular, 143 a large-diameter mode between $\sim 3-10$ microns was observed, which strongly indicates a non144 expiratory source. Owing to the extended volume of the singing mask, most participants could not 145 avoid having the mask rub against the sampling funnel while sampling from the bottom quadrant. 146 This strongly implicates shed fibers as a contributing particle source; consequently, the particle 147 emission rate from the bottom quadrant provides an upper limit to leakage of expired particles.

148 We obtain the overall mask efficiency $(\eta)$ for reduction of expiratory aerosol emission for each 149 individual while singing by combining the observed particle emission rates without a mask to 150 emission from the various quadrants plus through-mask (Figure 3b; see Methods). The median is $15198 \%$ and the mean $97 \pm 3 \%$. (If the measurements from the participant who repeated the activities 152 are combined into a single value the median is $97 \%$ and the mean $96 \pm 4 \%$.) Mask efficiencies of 153 greater than $93 \%$ were determined for all but two participants wearing the singing mask. One of 154 these individuals $(\eta=88 \%$ ) had the highest normalized emission rate for the top quadrant, 155 indicating that leakage around the nose contributed to this low, but still very good, efficiency. The 156 other individual $(\eta=91 \%)$ produced a somewhat high normalized emission rate from the mask 
157 top and the second highest from the mask bottom, suggesting that both leakage and, likely, shed 158 mask fibers contributed. Regardless, the observed high overall efficiencies indicate that with 159 proper fit, in particular ensuring a good seal around the nose, the singing mask provides substantial 160 reduction expiratory particle emission even while singing. Also, no visible spittle (i.e., droplets 161 much larger than those measured here) was observed with wearing the singing mask, suggesting efficient elimination of very large droplets.

163 The overall efficiency determined here for the singing mask greatly exceeds that found for 164 surgical masks, and further there is substantially less individual variation between participants 165 (Figure 3b,c). The singing mask design provides a tighter fit that reduces, but does not eliminate, 166 variability in the fit quality. This, together with the greater efficiency for air passing through the 167 mask, leads to the overall improved performance compared to a surgical mask. Notably, the overall 168 efficiency of the singing mask including leakage exceeds the efficiency of the surgical mask even 169 if we assume zero leakage flow. While some tools exist to help improve sealing of cloth and 170 surgical masks $(20,21)$ their use during singing is infeasible. Similarly, so-called double masking 171 has been suggested, including by the U.S. Centers for Disease Control (22), as a way to improve 172 both filtering ability and fit, and thus overall efficiency (23), but this too would not be appropriate 173 for singing. Even so, outside of singing contexts (e.g., public adoption), double masking does not 174 ensure one obtains a good seal and thus the actual benefit is difficult to know-especially given 175 the wide range of cloth masks available. Also, while qualitative, the participants generally 176 indicated that greater breathing comfort with the singing mask compared to the mask(s) each 177 individual typically wore, most likely owing to the increased mask-face separation. We speculate 178 that the more open design of the singing mask could increase mask wearing compliance.

179 While mask wearing plays an important role in reducing emission of expiratory particles, 180 masks can also reduce inhaled particles concentrations. To address this, we measured for one 181 participant the time-varying concentration of particles inside the singing mask during breathing 182 (Figure 4). When leaving the mask neck seal intentionally somewhat loose the peak particle 183 concentrations inside the mask upon inhalation approximately equaled the room air aerosol 184 concentration. Upon exhalation the concentration fell to very low values, indicative of the lower 185 aerosol concentration in exhaled breath compared to most ambient environments. However, when 186 the mask was worn as designed, with the neck seal tight, the inside-mask concentration upon 187 inhalation rose only to about $6 \%$ of the room air concentration, indicative of an effective efficiency 
of $94 \%$ for inhalation, similar to that found for expiratory particle emission. Distinct from expiratory aerosols, the room air aerosols were primarily smaller than 0.5 microns, with only $\sim 10 \%$ of the particles larger. Filtration efficiency typically increases with particle size above this range (24) and thus we expect the efficiency of the singing mask towards expiratory aerosols emitted by others will be even greater than found here. Similar to exhalation, the singing mask geometry likely contributes to the high filtration efficiency towards ambient particles during inhalation. The greater mask-face separation will lead to air being drawn through nearly the entirety of the mask surface area, with a consequently lower face velocity and increased filtration efficiency compared to conventional designs. Overall, this demonstrates that wearing of the singing mask provides major benefits to both the wearer and others.

In summary, the overall efficiency of the singing mask when fit properly compares favorably to that expected for N95 respirators. As the materials comprising the singing mask and N95s differ, the singing mask, or similarly designed masks, could provide a viable alternative to N95s outside of healthcare or other specialized occupational settings for situations when greater mask efficiency is necessary or desired. For singers and other performers, in particular, the singing mask, when fit properly, has sufficiently high efficiency - maintained throughout the act of singing - that its adoption could facilitate in-person rehearsals with multiple people as long as other best practices (e.g., good ventilation) are also adopted.

\section{Methods}

\section{Mask Design}

The singing mask, shown in Figure 1, uses a two-bone structure to separate the mask material from the main area of the face by about $6 \mathrm{~cm}$, while still allowing for a good seal. A $0.6 \mathrm{~cm}$ wide, $10 \mathrm{~cm}$ long thin aluminum strip is used around the nose, which the wearer can mold to their face. A felt strip on the inside runs across the nose area to help with sealing. The sides of the mask extend over the cheeks, nearly to the ears. Adjustable elastic ear loops keep the sides of the mask in place and two additional ties fasten the mask around the wearer's head to further seal the mask against the face. The mask completely envelops the wearer's jaw and chin, with an adjustable elastic band below the jaw that keeps the mask tight against the neck while allowing for free jaw movement. The mask has two main regions: the upper, boned structure that holds the filtering fabric in front of but away from the mouth and nose, and an unstructured, expanded volume below 
218 the chin. The upper region is composed of three layers in a cloth-liner-cloth arrangement, with 200

219 thread count cloth outer layers and and Pellon ${ }^{\circledR} 50$ inner layer attached to the cotton with a fusible 220 webbing material. The Pellon ${ }^{\circledR} 50$ inner layer helps to stiffen the mask material but likely provides 221 little filtering. The length of the top region is about $12 \mathrm{~cm}$. The lower expanded volume is made 222 of two cloth layers and opens at the bottom to allow for drinking by straw during rehearsals. The 223 opening is sealed by folding the mask twice and then securing with embedded Velcro strips. 224 Negligible particle emissions from this area were observed after closing. When closed, the length 225 of the expanded region is about $10 \mathrm{~cm}$. A modified version of the singing mask was also 226 constructed. The difference from the standard singing mask is that there is no bottom opening; the 227 modified mask is otherwise identical. During use, the cloth material comprising both the singing 228 and modified singing masks was observed to deflect inward (for inhalation) and outward (for 229 exhalation). However, the boning provides support that limits the amount of cloth deflection 230 associated with inhalation and exhalation. With intentionally loose wearing, the deflection 231 magnitude decreased. Hence, deflection provides a qualitative indication of good fit. The mask 232 internal volume is about $0.5 \mathrm{~L}$. This is similar to the tidal volume associated with normal breathing 233 (25), but about half that for singing (26). Given the limited deflection of the mask material this 234 implies substantial exchange of air, which will help to alleviate any depletion of oxygen or buildup 235 of $\mathrm{CO}_{2}$.

236 Proper wearing of the singing masks includes: first securing the mask using the ear loops, 237 molding the aluminum strip around the nose, tightening the ear loops, tightening the neck strap 238 elastic band, tying the top strap around the users head near the parietal eminence, and then tying 239 the bottom strap around the users neck while sitting or standing up straight. With proper wearing, 240 one should see no obvious gaps, especially around the nose; this can be qualitatively assessed by 241 having the wearer look down towards their nose moving only their eyes. If they can see their nose 242 below the mask then there is a gap and the mask should be better secured.

243 Two additional masks were constructed using the same materials as the singing mask. One was 244 constructed having only two cloth layers and one having three layers (cloth-liner-cloth). Both used 245 a general pleated surgical mask design, based on the design initially promoted by the U. S. Centers 246 for Disease Control; the directions and instructional video originally made available by the CDC 247 are no longer available online. Both used the same ear loops as with the singing mask and included 248 two head straps. Additional tests for the through-mask efficiency were performed by one 
participant using an N95 respirator (3M, Model 8210), two different surgical masks (a medical-

250 grade ValuMax 5130E-SB and an unknown model), and a non-medical 'Fashion Dust Preventive 251 Mask' (30\% cotton, 70\% polyester) from YiWu Xuefeng Mask Factory, both without (FDPM) and 252 with (FDPM(N95)) an N95 insert.

\section{Human subjects}

We recruited 12 volunteers (4 self-identified male and 8 self-identified female), ranging in age from 18 to 65 years old. The Institutional Review Board of the University of California, Davis approved this work (IRB\# 844369-4), and all research performed followed the Institutional Review Board guidelines and regulations. Prior to the tests, written informed consent was obtained from all participants. Information collected from participants included their age and singing range (e.g., soprano, alto, baritone). Only self-reported healthy non-smokers were included in the study. All 260 participants had to take the UC Davis Daily Symptom Survey 261 (https://campusready.ucdavis.edu/symptom-survey) prior to accessing campus. Participants were encouraged to obtain a negative COVID-19 test just prior to their participation, although this was not required or tracked. Informed consent for publication of identifying information was obtained

264 from the participant shown in Figure 1.

\section{Expiratory Aerosol Experimental Description}

We used an experimental setup similar to that in previous work $(9,10,27)$. In brief, participants were asked to breath, speak, or sing in front of a stainless steel funnel connected by nonconductive tubing to both an aerodynamic particle sizer (APS, TSI Model 3321, $5 \mathrm{~L} / \mathrm{min}$ ) and a condensation particle counter (CPC, TSI Model 3775, $0.3 \mathrm{lpm}$ ) that were located in a HEPA-filtered laminar flow hood. The APS characterizes particles from 0.3 to 20 microns in aerodynamic diameter, with a decreased detection efficiency for particles $<0.5$ microns. The CPC characterizes the number

272 concentration of all particles sampled, although with a reduced efficiency for particles larger than 273 about 1 micron owing to impaction losses.

274 Participants donned the singing mask without direct assistance. They were asked to tighten 275 the ear loops and the neck closure, pinch closed the metal bar in the singing mask around their 276 nose, and to tie the neck and head straps. They were asked to "tighten everything as much as 277 possible, but such that you are still comfortable." 
Respiratory emissions with or without a mask were tested with the participant's head oriented in one of four positions, relative to the sampling funnel. These orientations were the same as those described in Cappa et al. (11) and are shown in Figure S3. These were as follows.

(i) Forward/Through: The participants sat directly facing the APS funnel. This was the orientation examined in prior studies $(9,10,27)$. In this orientation, the APS samples air that has passed through the mask material.

(ii) Top: The participants tilted their heads downward to have the bridge of the nose approximately centered on the APS funnel, allowing for sampling of particles that leak from the mask nose area.

(iii) Side: The participants turned their head 90 degrees to face perpendicular to the APS funnel, with the side singing mask approximately centered on the funnel

(iv) Bottom: The participants positioned their chin just above the APS funnel with the mask material from the expanded volume over the top of the funnel. This allowed for sampling of particles that leak from the mask neck area.

294 were asked to read the "Rainbow Passage," both with no mask and while wearing the mask while 295 oriented in the "forward" direction (Figure S3). Participants also performed two singing activities. 296 First, they sang in English Beethoven's Ode to Joy from his Ninth Symphony, both wearing and 297 not wearing the mask in each of four head orientations described above (Figure S3). Second, 298 participants sang a song of their choosing of about two minutes in length. They performed this second activity both without a mask and with the mask in the forward orientation only.

300 For all speaking and singing activities, participants were asked to carry out the activity at a 301 comfortable volume; no effort was made to control for volume differences between participants. 302 While loudness can influence the emission rate of expiratory aerosols (9), we focus on the 303 reduction achieved by wearing the mask, and thus loudness differences will have little effect. All 304 particle emission rates were adjusted to units of particles per second by accounting for the actual 305 duration of vocalization $\left(t_{\mathrm{voc}}\right)$, which excludes pauses between words or phrases as determined 
306 from microphone recordings. One participant repeated the Ode to Joy activities multiple times on 307 different days.

308 The directly observed particle emission rates $\left(\dot{N}_{p}^{o b s}\right)$ does not necessarily equate to the total 309 particle emission rate owing to differences between the APS total airflow rate (5 lpm) and the 310 airflow rate of the expiratory activity ( $Q_{\text {exp,tot }}$ ), as discussed in Cappa et al. (Submitted).(11) This 311 raises certain challenges when combining the measurements from the different orientations to 312 estimate the overall mask efficiency. Typical airflow rates associated with talking range from ca. $3138-15 \mathrm{lpm}(16)$. For singing, airflow rates are in the same general range although skewed perhaps a 314 little higher, especially for louder singing, and females tend to exhibit slightly smaller values than 315 males $(28,29)$. Consequently, the actual particle emission rates associated with talking and singing 316 without a mask are about a factor of 8-15 times higher than the observed values. We present the 317 unadjusted (observed) absolute values to remain consistent with previous studies.

318 With mask wearing the airflow during expiration can be split in multiple directions, with the 319 amount of air in a given direction not known a priori. We previously accounted for this split for 320 surgical masks while talking or coughing using a Monte Carlo method to determine probability 321 distributions of the overall mask efficiency based on the median values across the population of 322 participants (11). Overall, relatively narrow probability distributions resulted with only moderate 323 sensitivity to the assumed split between the air that passed through the mask versus escaped out 324 the top, sides, or bottom and the greatest deviations found for very low total expiratory airflow 325 rates. We used a similar approach here, but applied the approach to the observations from each 326 individual, rather than using the medians across participants. Over 10,000 iterations, we 327 determined the fraction $\left(f_{\mathrm{x}}\right)$ of air that goes in a particular direction from a random distribution. 328 We further assumed a log-normal distribution of expiratory airflow rates centered at $13 \mathrm{lpm}$ with 329 a width of 1.3. The $\dot{N}_{p, i}^{o b s}$ in each orientation (i) for each individual are adjusted to actual particle 330 emission rates $\left(\dot{N}_{p, i}\right)$ based on the above assumptions. While we present the unadjusted (observed) 331 absolute values to remain consistent with previous studies, when reporting particle emission rates 332 normalized to the no-mask condition we use the airflow-adjusted values, which are also used to 333 calculate the overall mask efficiency. The overall mask efficiency, $\eta$, is:

$$
\eta=1-\frac{\sum \dot{N}_{p, i}}{\dot{N}_{p, \text { nomask }}}
$$


335 The average value and standard deviation for each individual were determined from the 336 distribution of $\eta$ values from the simulations.

337 In the "bottom" orientation, the participants positioned their chin just above the APS funnel, 338 with the mask material from the expanded volume draped over the top of the funnel. Some 339 participants could not completely avoid contact between the mask material and the funnel in this 340 position; consequently, mask fibers shed by friction between mask and funnel may have 341 contributed to the particle counts from participants in this orientation. $(10,30,31)$ Such non-

342 expiratory particles confound the respiratory emission measurements, but they may still carry 343 pathogens as aerosolized fomites (32). Based on the observed particle size distributions, a few 344 participants appeared to generate a significant amount of mask-fiber particles. For these 345 participants, when assessing the overall mask efficiency, we used the median value from the other 346 participants in place of the value measured for the individual.

347 One participant sang Ode to Joy wearing a variety of mask types (see Mask Design) in the 348 forward (through-mask) position, with three replicates for each mask type.

349 All data processing analyses were carried out using Igor Pro (v. 8.0.4.2, Wavemetrics).

350 Differences between the $\dot{N}_{p, i}$ values are calculated after log-transformation using a single factor 351 ANOVA test. breathing. Here, a tube composed of conductive silicon was inserted below the mask at the neck area and the sampling end of the tube was positioned to sit in the main mask area in front of the face. The tube was attached to a condensation particle counter, which sampled at $0.3 \mathrm{lpm}$ and measured the total concentration of particles every 1 second. Two experiments were conducted. In both, the participant was asked to breathe deeply in and out through their nose 10 times at a rate of about five breaths per minute while the particle concentration inside the mask was continually 361 the singing mask. For the second, the neck strap was left slightly loose to intentionally introduce 362 a leak. Prior to starting the measurement, the participant was asked to breathe three times after the 363 sampling tube was inserted. The concentration of particles in the room air was measured just prior to the measurements of particle concentrations inside the mask. 
366 For an expiratory jet, the air velocity, $u$, decreases with distance as $1 /(\alpha \cdot x)$, where $\alpha$ is the 367 divergence angle $\left(\sim 20^{\circ}\right)$ and $x$ is distance $(17)$. Single-fiber filtration efficiencies $\left(\eta_{f}\right)$ increase as 368 velocity decreases, with $\eta_{f} \sim 1-\exp \left(K \cdot u^{-\frac{2}{3}}\right)$, and where $K$ is a scaling factor (33). Thus, for 369 an aerosols carried in an expanding jet the single-fiber filtration efficiency will scale with the 370 mouth-mask material separation distance as $\eta_{f} \sim 1-\exp \left(K^{*} \cdot x^{\frac{2}{3}}\right)$, where $K^{*}$ is a scaling factor.

371 For a mask-face separation distance of $\sim 6 \mathrm{~cm}$ for the singing mask, compared to $\sim 0.5 \mathrm{~cm}$ for a 372 conventional cloth mask, the air velocity through the singers mask is reduced by about $73 \%$, 373 leading to an increase in the single-fiber filtering efficiency (Figure S6). For an inspiratory flow, 374 the airflow may pass through a greater proportion of the overall mask surface area when the mask 375 surface is sufficiently separated from the face to avoid mask-face contact and when leakage is 376 negligible. The face velocity for the singing mask relative to a conventional cloth mask that makes 377 contact with the face will scale as the mask area through which the air passes. The total mask area 378 is about $550 \mathrm{~cm}^{2}$. For a conventional mask, we assume that the airflow passes through a circular 379 area around the nose and the mouth, and we estimate a typical diameter will be $\sim 7 \mathrm{~cm}$, 380 corresponding to an area of $38 \mathrm{~cm}^{2}$. Thus, the face velocity through the singers mask during 381 inspiration is $\sim 14$ times as large as through a conventional cloth mask, which engenders a large 382 increase in the single-fiber filtration efficiency (Figure S6). 


\section{Reference}

385 1. J. Howard et al., An evidence review of face masks against COVID-19. Proceedings of

2. J. T. Brooks, J. C. Butler, Effectiveness of Mask Wearing to Control Community Spread of SARS-CoV-2. JAMA, (2021).

3. T. Dai, G. Bai, G. F. Anderson, PPE Supply Chain Needs Data Transparency and Stress Testing. Journal of General Internal Medicine 35, 2748-2749 (2020).

4. Public Health England, Investigation of novel SARS-CoV-2 variant, Variant of Concern 202012/01; Technical briefing 2 (2021 https://assets.publishing.service.gov.uk/government/uploads/system/uploads/attachment data/file/948152/Technical_Briefing_VOC202012-2_Briefing_2 FINAL.pdf).

5. J. L. Scheid, S. P. Lupien, G. S. Ford, S. L. West, Commentary: Physiological and Psychological Impact of Face Mask Usage during the COVID-19 Pandemic. International Journal of Environmental Research and Public Health 17, 6655 (2020).

6. S. Taylor, G. J. G. Asmundson, Negative attitudes about facemasks during the COVID19 pandemic: The dual importance of perceived ineffectiveness and psychological reactance. medRxiv, 2020.2011.2017.20233585 (2020).

7. S. L. Miller et al., Transmission of SARS-CoV-2 by inhalation of respiratory aerosol in the Skagit Valley Chorale superspreading event. Indoor Air 31, 314-323 (2021).

8. M. Alsved et al., Exhaled respiratory particles during singing and talking. Aerosol Science and Technology 54, 1245-1248 (2020).

9. S. Asadi et al., Aerosol emission and superemission during human speech increase with voice loudness. Scientific Reports 9, 2348 (2019).

10. S. Asadi et al., Efficacy of masks and face coverings in controlling aerosol particle emission from expiratory activities. Scientific Reports 10, 15665 (2020).

11. C. D. Cappa et al., Expiratory aerosol particle escape from surgical masks due to imperfect sealing. Scientific Reports, (Submitted).

12. B. Lindblom, J. Sundberg, in Springer Handbook of Acoustics, T. Rossing, Ed. (Springer, New York, NY, 2007).

13. H. J. Rubin, M. LeCover, W. Vennard, Vocal Intensity, Subglottic Pressure and Air Flow Relationships in Singers. Folia Phoniatrica et Logopaedica 19, 393-413 (1967).

14. G. Fairbanks, Voice and Articulation Drillbook. (Harper and Row, New York, ed. 2nd, 1960), pp. 234.

15. R. Saeidi, I. Huhtakallio, P. Alku, in INTERSPEECH 2016. (International Speech Communication Association, San Francisco, 2016), pp. 1800-1804.

16. J. K. Gupta, C.-H. Lin, Q. Chen, Characterizing exhaled airflow from breathing and talking. Indoor Air 20, 31-39 (2010). 
17. M. Abkarian, S. Mendez, N. Xue, F. Yang, H. A. Stone, Speech can produce jet-like transport relevant to asymptomatic spreading of virus. Proceedings of the National Academy of Sciences 117, 25237-25245 (2020).

18. T. Oberg, L. M. Brosseau, Surgical mask filter and fit performance. American Journal of Infection Control 36, 276-282 (2008).

19. S. A. Grinshpun et al., Performance of an N95 Filtering Facepiece Particulate Respirator and a Surgical Mask During Human Breathing: Two Pathways for Particle Penetration. Journal of Occupational and Environmental Hygiene 6, 593-603 (2009).

20. D. A. Rothamer, S. Sanders, D. Reindl, T. H. Bertram, Strategies to minimize SARSCoV-2 transmission in classroom settings: Combined impacts of ventilation and mask effective filtration efficiency. medRxiv, 2020.2012.2031.20249101 (2021).

21. P. W. Clapp et al., Evaluation of Cloth Masks and Modified Procedure Masks as Personal Protective Equipment for the Public During the COVID-19 Pandemic. JAMA Internal Medicine, (2020).

22. J. T. Brooks et al., Maximizing Fit for Cloth and Medical Procedure Masks to Improve Performance and Reduce SARS-CoV-2 Transmission and Exposure. MMWR Morb Mortal Wkly Rep, (2021).

23. M. Gandhi, L. C. Marr, Uniting Infectious Disease and Physical Science Principles on the Importance of Face Masks for COVID-19. Med 2, 29-32 (2021).

24. F. Drewnick et al., Aerosol filtration efficiency of household materials for homemade face masks: Influence of material properties, particle size, particle electrical charge, face velocity, and leaks. Aerosol Science and Technology 55, 63-79 (2021).

25. S. Hallett, F. Toro, J. V. Ashurst, Physiology, Tidal Volume, in StatPearls [Internet] (StatPearls Publishing, Treasure Island, FL, 2020), Accessed on: 2 February 2021, https://www.ncbi.nlm.nih.gov/books/NBK482502/.

26. B. Binazzi et al., Breathing pattern and kinematics in normal subjects during speech, singing and loud whispering. Acta Physiologica 186, 233-246 (2006).

27. S. Asadi et al., Effect of voicing and articulation manner on aerosol particle emission during human speech. PLoS One 15, e0227699 (2020).

28. J. J. Jiang, R. B. Hanna, M. V. Willey, A. Rieves, The Measurement of Airflow Using Singing Helmet That Allows Free Movement of the Jaw. Journal of Voice 30, 641-648 (2016).

29. E. B. Holmberg, R. E. Hillman, J. S. Perkell, Glottal airflow and transglottal air pressure measurements for male and female speakers in soft, normal, and loud voice. The Journal of the Acoustical Society of America 84, 511-529 (1988).

30. D. Hospodsky et al., Human Occupancy as a Source of Indoor Airborne Bacteria. PLoS One 7, e34867 (2012).

31. R. P. Clark, S. G. Shirley, Identification of Skin in Airborne Particulate Matter. Nature 246, 39-40 (1973). 
460 32. S. Asadi et al., Influenza A virus is transmissible via aerosolized fomites. Nature

$461 \quad$ Communications 11, 4062 (2020).

462 33. K. W. Lee, B. Y. H. Liu, Theoretical Study of Aerosol Filtration by Fibrous Filters.

$463 \quad$ Aerosol Science and Technology 1, 147-161 (1982).

\section{Acknowledgements}

466 The authors greatly appreciate the participation of the various professional and amateur singers 467 who volunteered their time. Author contributions: C. D. C. led the measurements and analysis. 468 S. A. R. and A. A-K.. of the San Francisco Opera, SFO) designed the mask. SFO constructed the 469 masks and SFO and C. D. C provided design suggestions. W. D. R. contributed to experimental 470 design and interpretation. A. S. W., S. B., and N. M. B. contributed to interpretation. C. D. C, and 471 S. A. R. led the manuscript writing. All authors reviewed and revised the manuscript for accuracy 472 and intellectual content. Competing interests: S. A. R. and A. A-K., have filed a patent application 473 for the singing mask. All other authors declare no competing interests. Data and materials 474 availability: All data needed to evaluate the conclusions in the paper are present in the paper 475 and/or the Methods. Additional data and files related to this paper will be made publicly available 476 at doi:10.25338/B8GD1B and are available for review at https://bit.ly/3qUJpiy. 

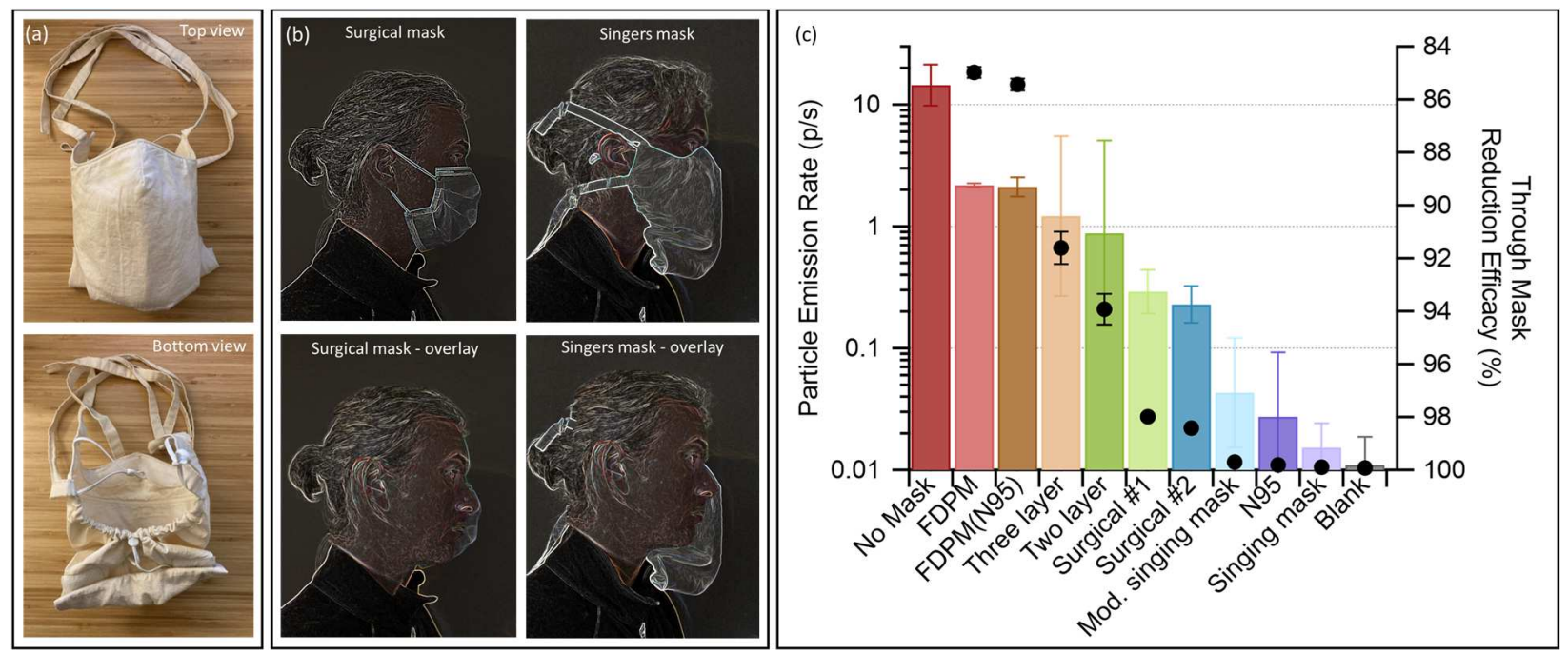

Figure 1. (a) Top and bottom view of the singing mask. (b) (top row) Images of one participant wearing a surgical mask and wearing the singing mask. (bottom row) Overlaid images of the participant with the surgical mask and with a singing mask to illustrate the increased mask-face material separation in the singing mask. The images in (b) were modified from the originals using the "glow edges" artistic effect in Microsoft PowerPoint to accentuate the mask edges and to overlay an image of the participant with no mask. Informed consent was obtained. (c) Observed particle emission rate for one participant singing Ode to Joy with no mask, or a homemade cloth two-layer or three-layer pleated mask made of the same material as the singing mask, a modified singing mask having no bottom opening, the standard singing mask (purple), a commercial cloth mask either without (FDPM) or with (FDPM(N95)) an N95 insert, either of two different surgical masks, or an N95 respirator (3M), as measured in the forward (throughmask) position. Note that jaw movement was substantially restricted with the non-singing masks, especially the N95 respirator. Three repeats were performed and error bars are $1 \sigma$ geometric standard deviations. The right axis gives the associated reduction efficiency for the forward

494 direction (black points). 


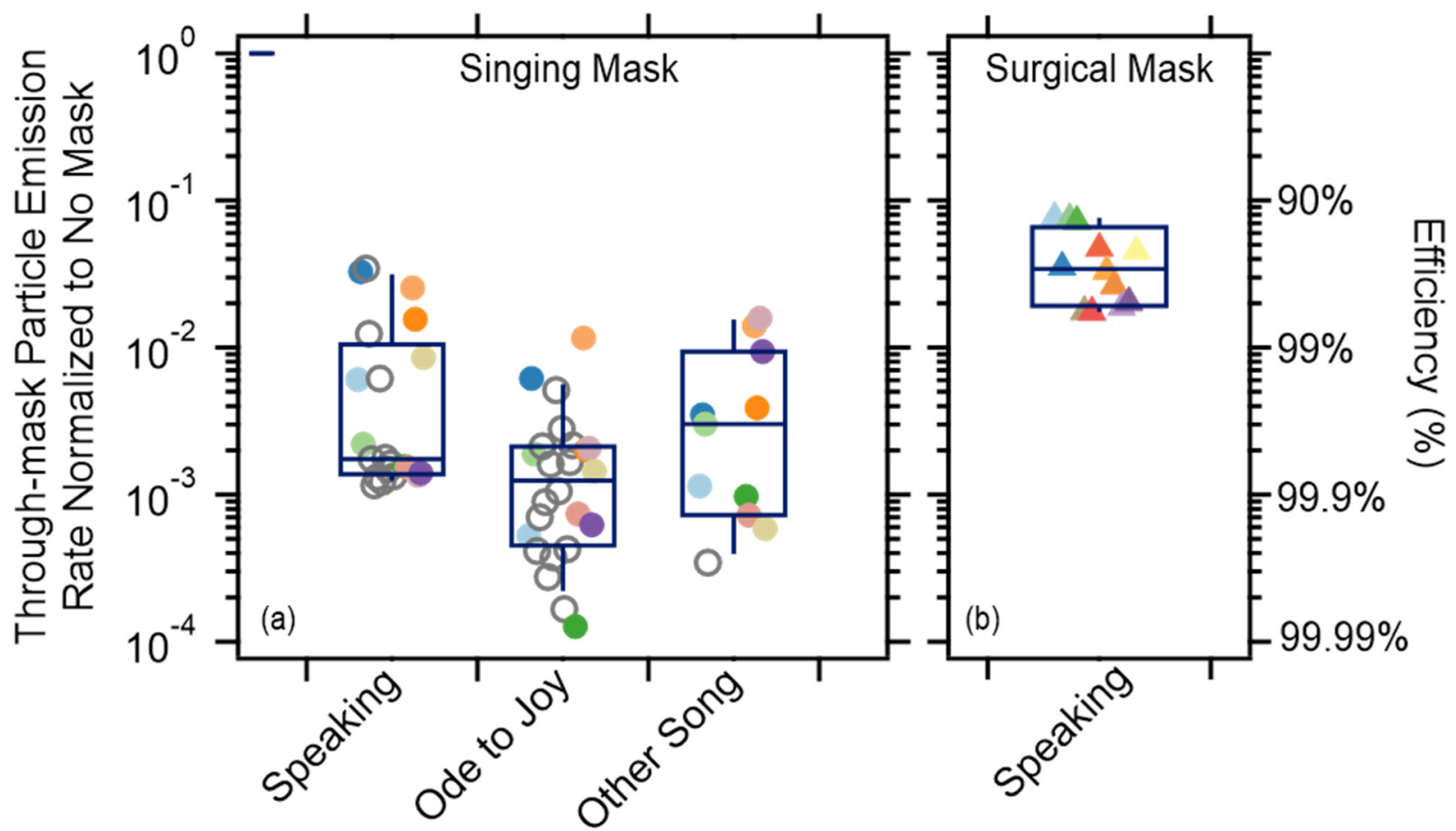

498 Figure 2. (a) The through-mask (forward) particle emission rates normalized to no mask (left 499 axis) or the corresponding reduction efficiency (right axis) observed for participants while 500 wearing the singing mask for speaking or singing either the Ode to Joy or a song chosen by the 501 participant. Closed colored circles in (a) indicate unique individuals while open gray circles 502 indicate repeats by one individual. (b) Normalized particle emission rates for participants 503 speaking while wearing a surgical mask (data from Cappa et al. (11)). Note that the participants 504 in (a) differ from those in (b). Box and whisker plots show the median, $25^{\text {th }} / 75^{\text {th }}$ percentile, and $50510^{\text {th }} / 90^{\text {th }}$ percentile. 

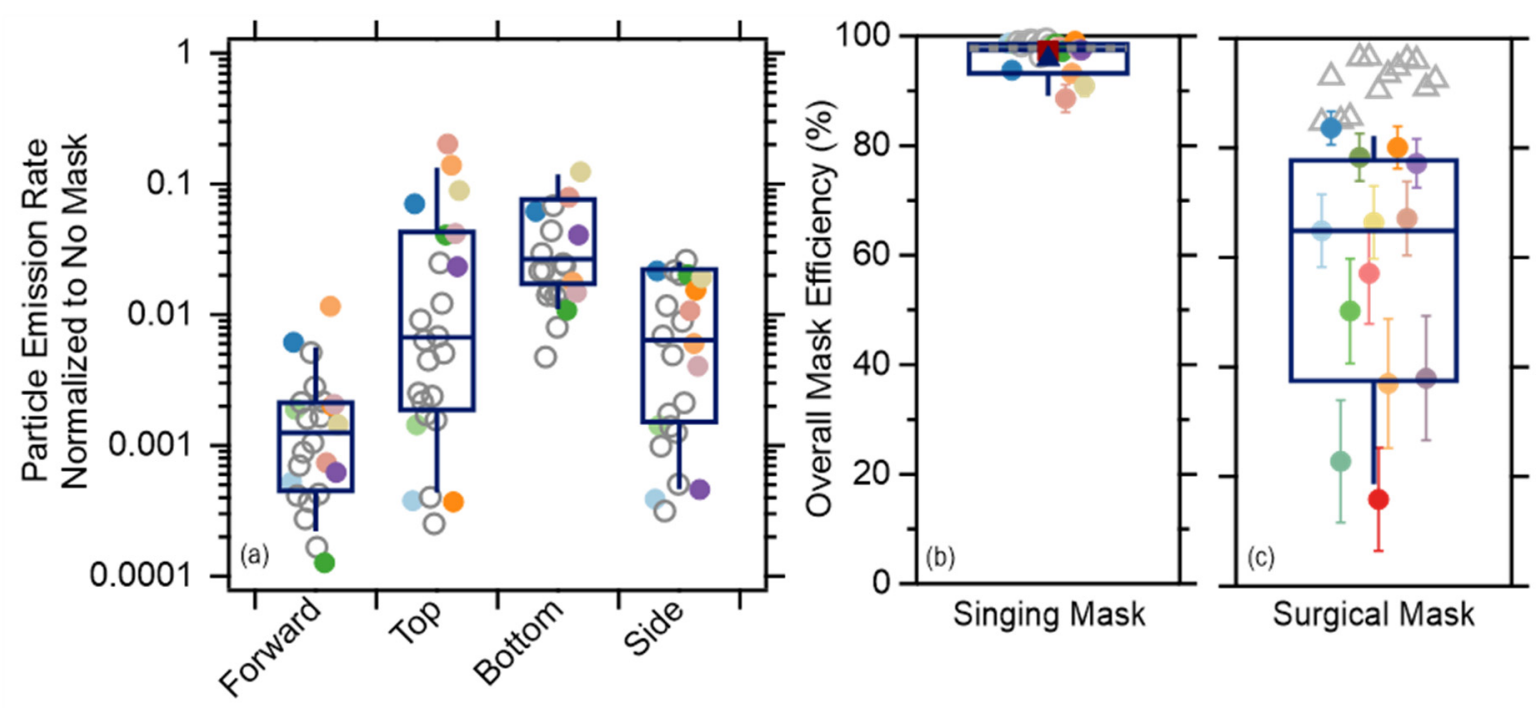

Figure 3. (a) Particle emission rates normalized to no mask wearing for singing Ode to Joy for the different orientations while wearing the singing mask. (b) The overall mask efficiency while singing Ode to Joy with the singing mask, accounting for leakage flows, colored by participant

512 (circles). Uncertainty bars on individual points indicate $1 \sigma$ determined from Monte Carlo

513 simulations. The red square is the average across all measurements and the blue triangle the

514 average after combining replicate measurements from one participant. (c) Overall efficiency for

515 speaking with a surgical mask including leakage flows (solid points) or assuming all flow passes 516 through the mask, i.e. zero leakage flows and perfect sealing (open points). Closed circles in (b) 517 and (c) indicate unique individuals while open circles indicate repeats by one individual. Note 518 that the participants in $(a, b)$ differ from those in (c). Box and whisker plots show the median, $51925^{\text {th }} / 75^{\text {th }}$ percentile, and $10^{\text {th }} / 90^{\text {th }}$ percentile. 


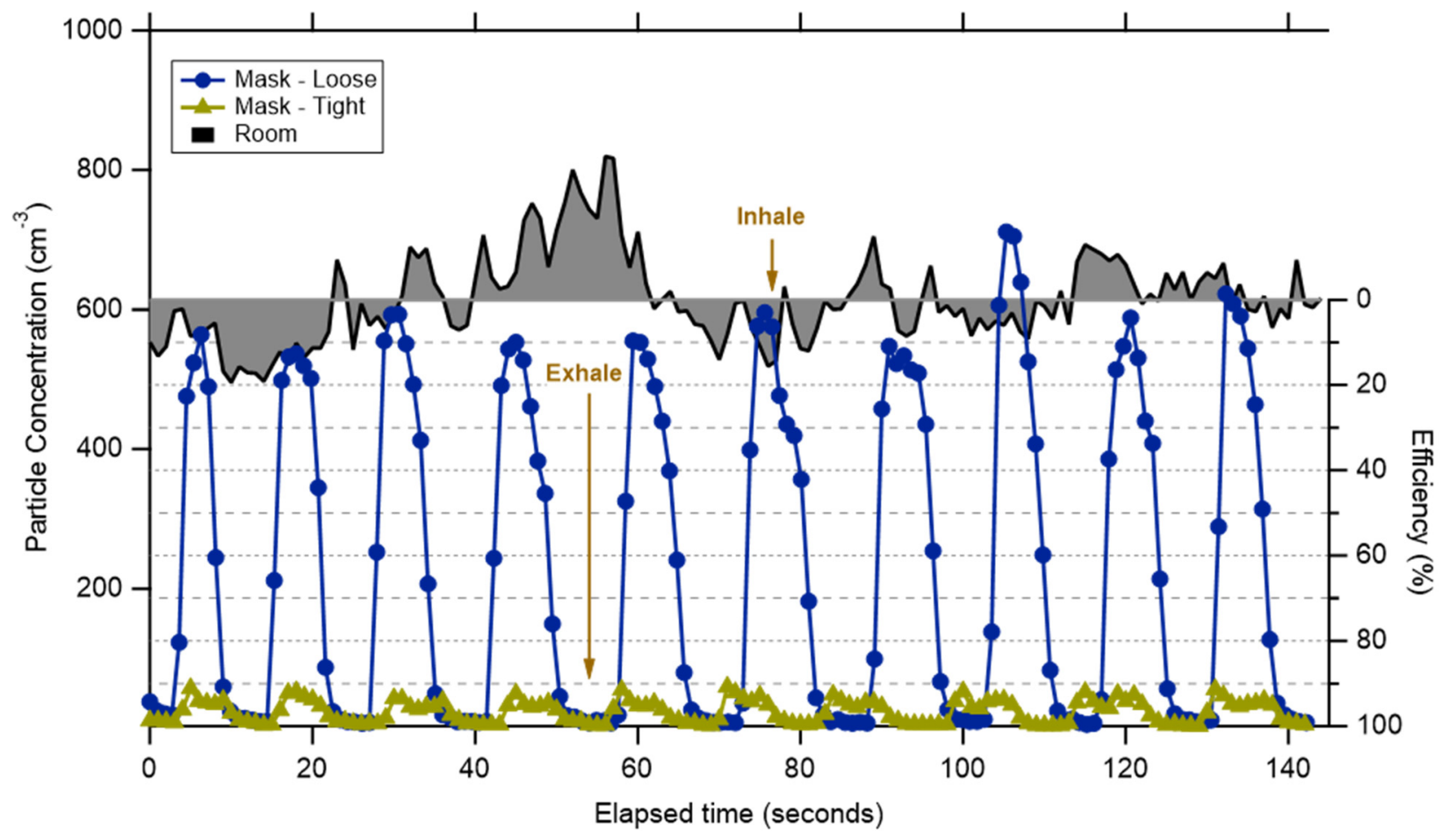

Figure 4. Time-series of particle concentrations measured for room air (solid black line), sampled 523 inside the singing mask worn intentionally loose around the neck (blue circles), and when sampled 524 inside the mask when worn tightly as designed (gold triangles). The right axis shows the 525 approximate mask efficiency based on comparison with the mean room air concentration. 


\section{Extended Data Figures}

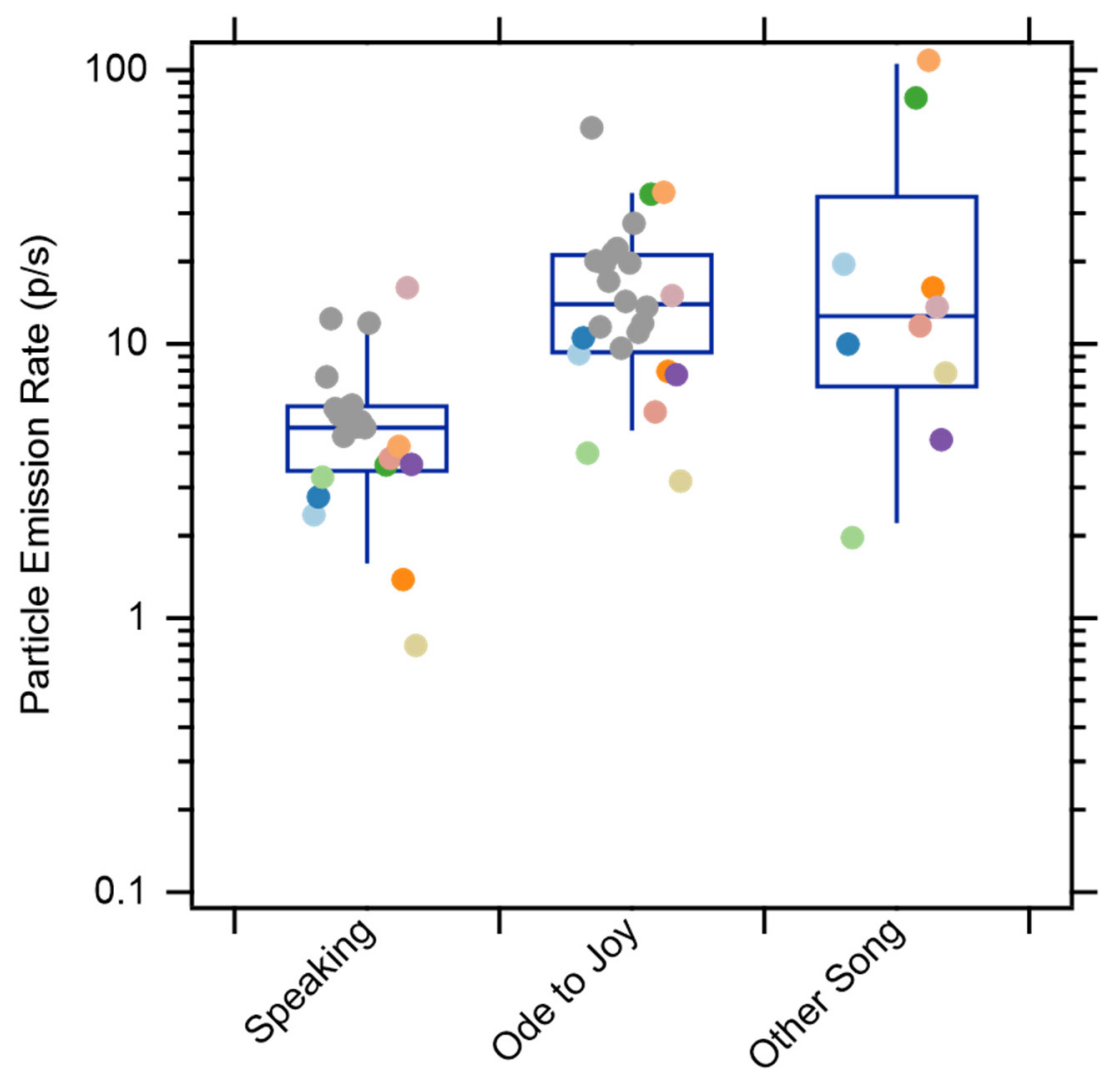

531 Figure S1. Observed particle emission rates (p/s) for the individual participants without mask 532 wearing. These have not been corrected for undersampling by the APS (see methods). Emissions 533 from the singing activities are three times greater than for speaking; the difference is statistically 534 significant $(p=0.002)$. Points are colored by participant, with the gray dots indicating repeats from 535 one individual. 

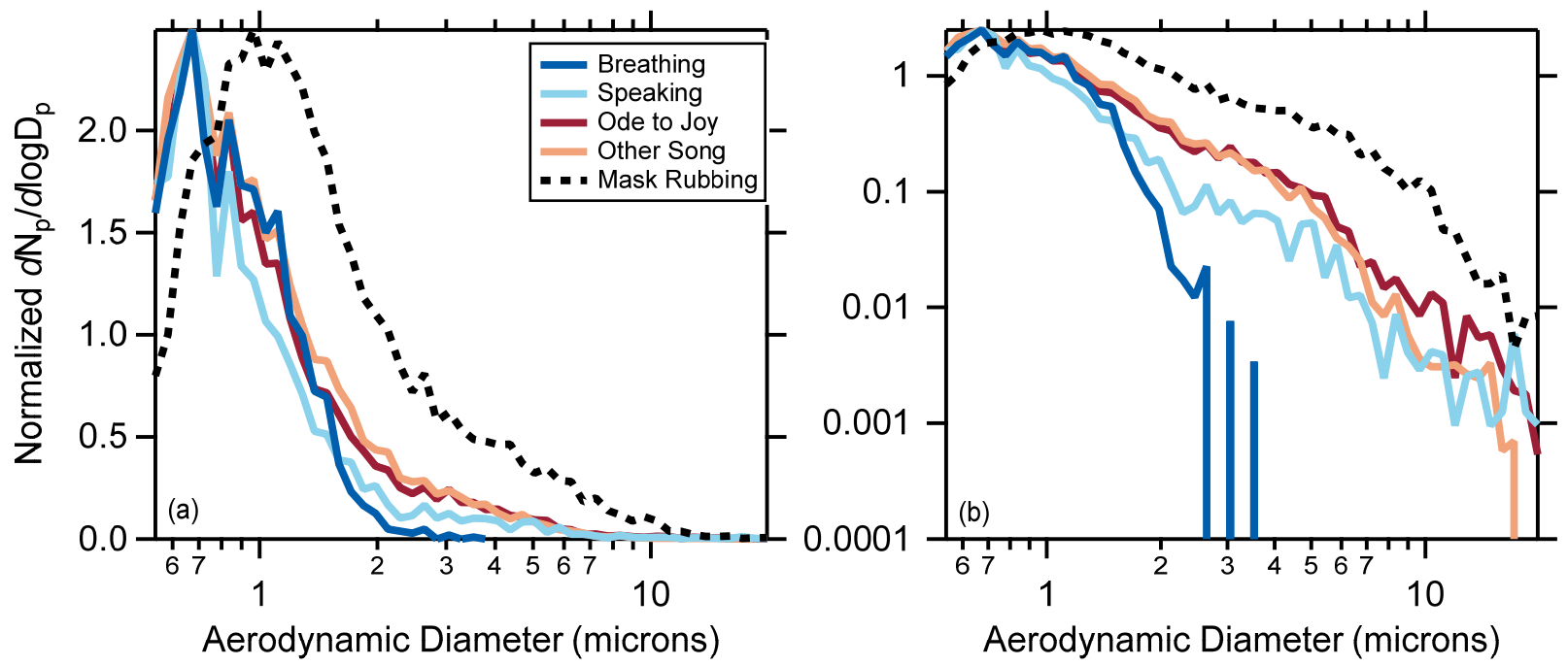

Figure S2. Particle emission rate size distributions for various expiratory activities (solid lines),

540 i.e., breathing, speaking, and singing either the Ode to Joy or a song of the participants choice, and

541 the size distribution resulting from physical rubbing of the mask material against itself (dashed

542 line). All distributions are averages across the participants. The distributions are shown on (a) 543 linear or (b) logarithmic y-axes. 
(a)

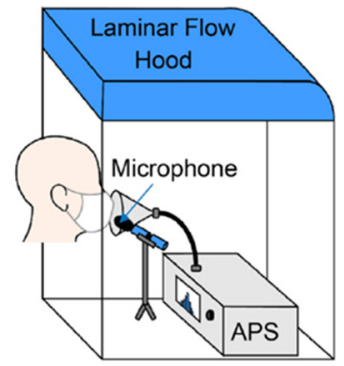

(b)

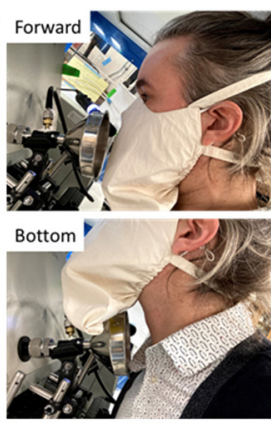

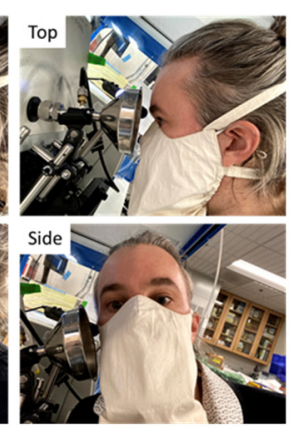

(c)

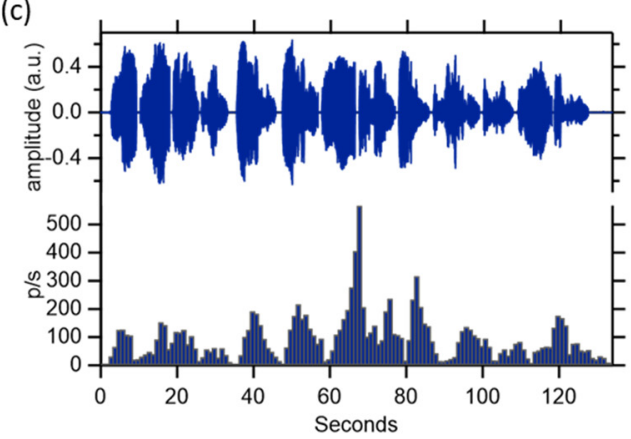

545

546

547

548

549

550

551

Figure S3. (a) Schematic of the experimental setup showing a participant wearing a mask in the forward orientation. (b) Photographs showing a participant in each of the four sampling orientations: forward, top, side, and bottom sampling. (c) Example microphone recording for a participant singing a song of the participants' choice without a mask and the associated particle counts by the APS. 

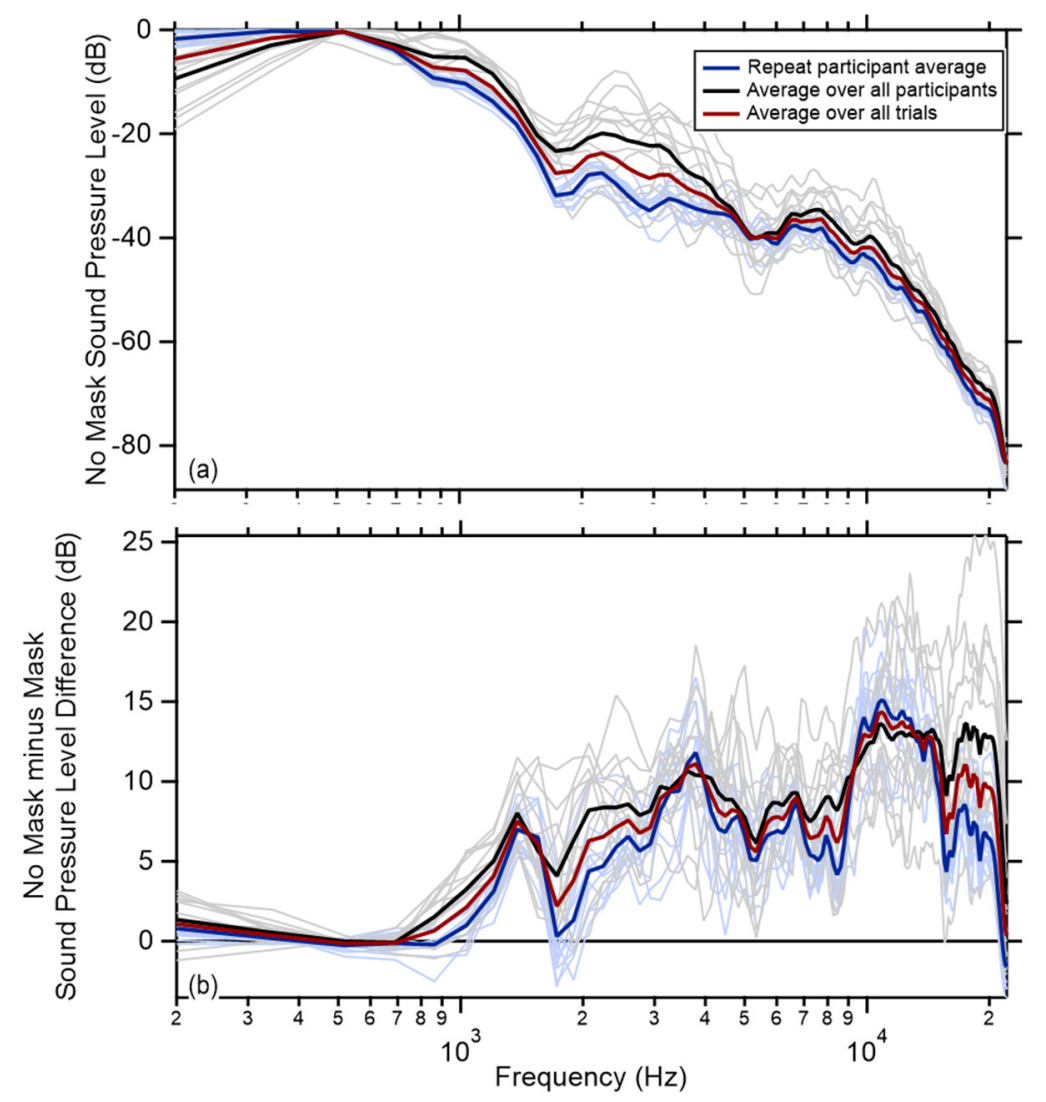

554 Figure S4. (a) Average power spectrum with no mask, set to full scale, and (b) difference between 555 no mask and mask for participants singing Ode to Joy. Thin lines are individual measurements, 556 with blue lines for the participant who performed replicate trials. The solid lines are averages 557 across participants for (red) all individual trials, (blue) the repeat performer, and (black) over all 558 participants using the average from the repeat performer. 


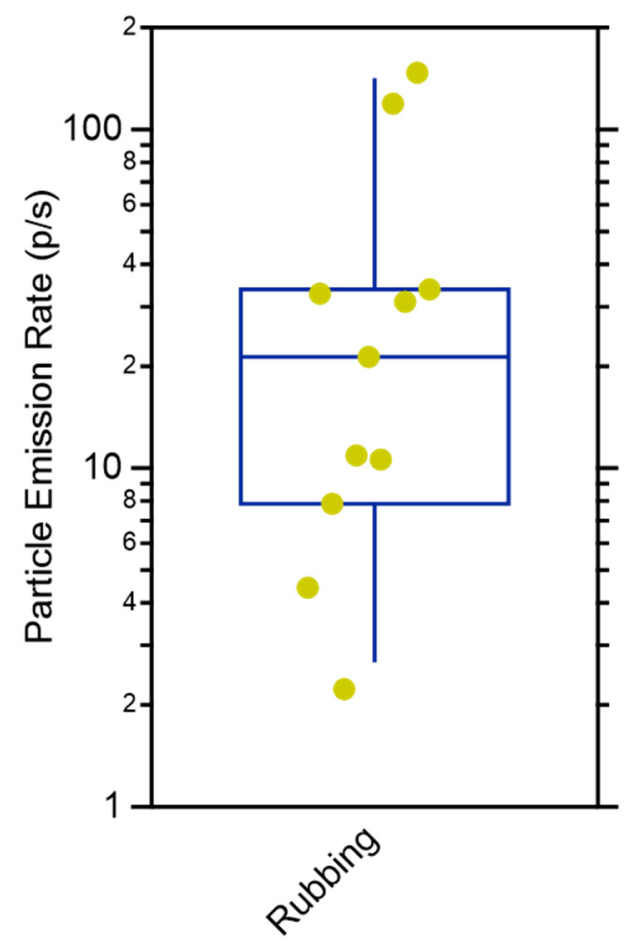

562 Figure S5. Observed particle emission rates $(\mathrm{p} / \mathrm{s})$ for rubbing the singing mask material together. 563 


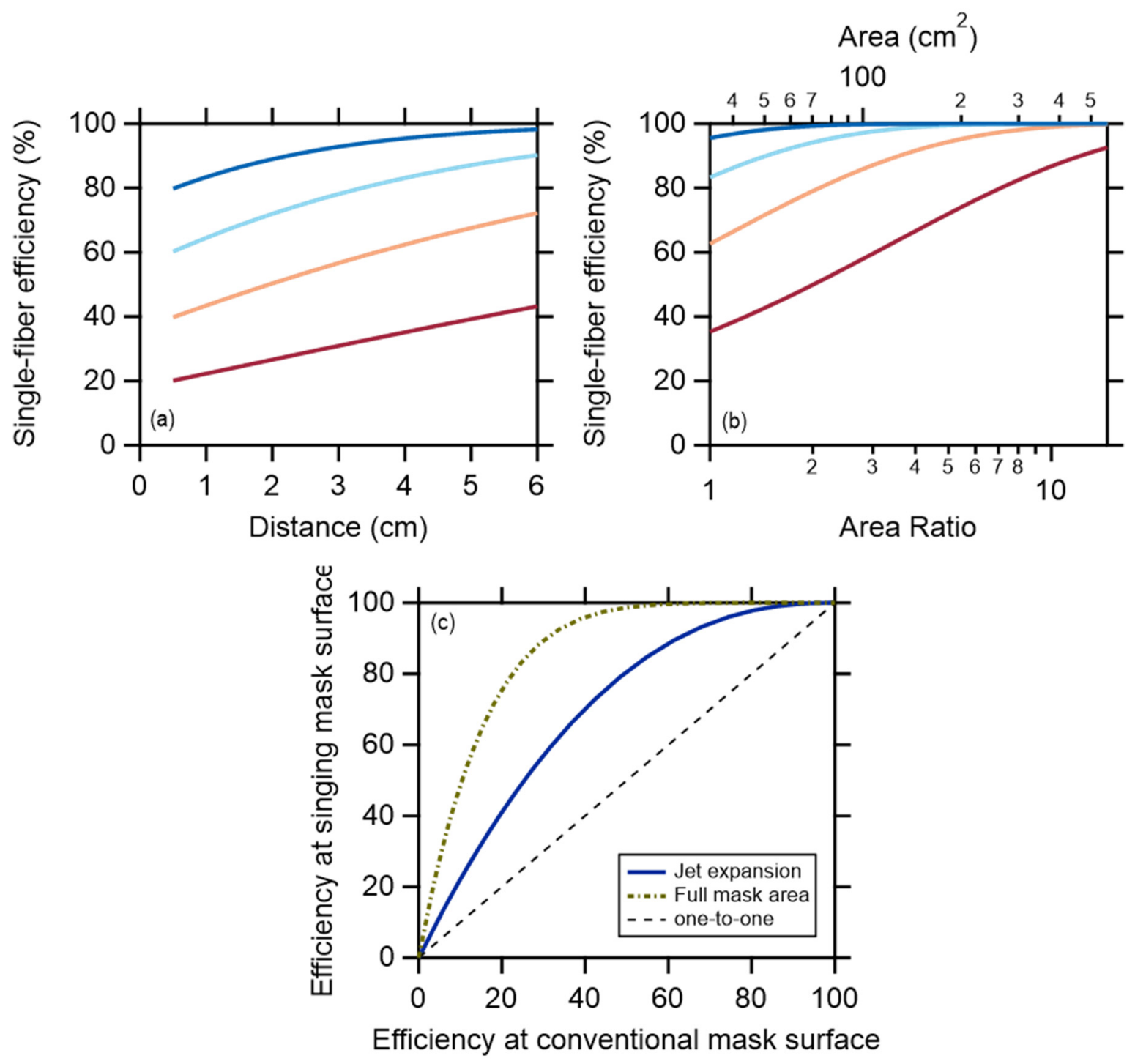

566 Figure S6. Effect of increasing the distance between the mouth and the mask material on singlefiber filtration efficiency. (a) Relationship between single-fiber efficiency and distance assuming an expanding conical jet that diverges at an angle of $20^{\circ}$ with a radius of $2 \mathrm{~cm}$ at a distance of 0.5 , which approximates expansion of an expiratory airflow. (b) Relationship between single-fiber efficiency and the mask surface area ratioed to the area of a circular area around the mouth and nose with a radius of $3.5 \mathrm{~cm}$. Four curves are shown to illustrate how efficiency varies depending on the assumed efficiency at the reference distance or area. (c) The relationship between the efficiency at the singing mask surface and the expected efficiency at the surface of a conventional mask assuming an expanding conical jet (as in (a); blue line) or for the total mask area (as in (b); gold line). The black dashed line is the one-to-one line. The decreased air velocity at the mask surface engenders a large increase in the single-fiber filtering efficiency. 

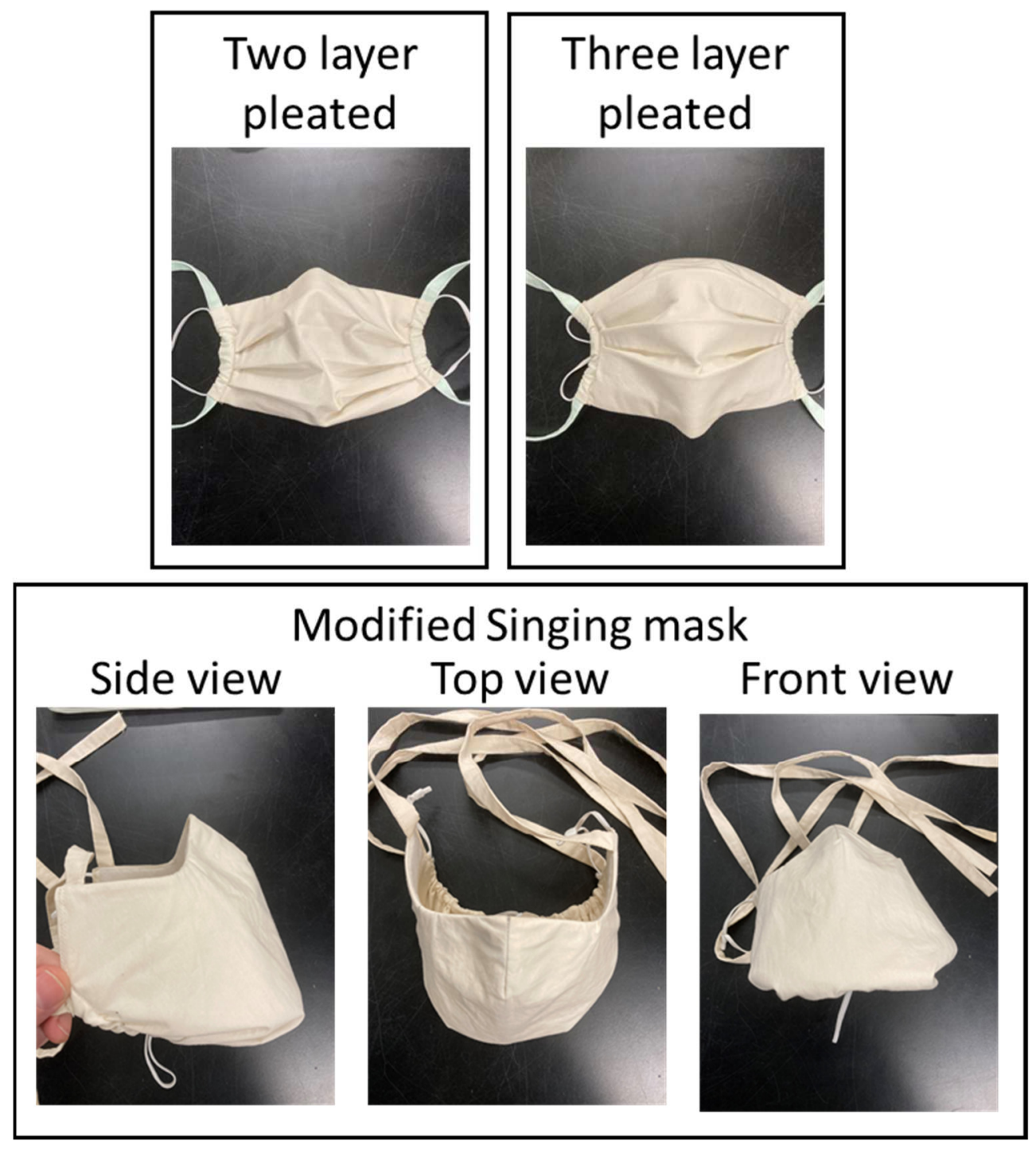

579 Figure S7. Photographs of the two-layer and three-layer pleated masks and the modified singing 580 mask. 


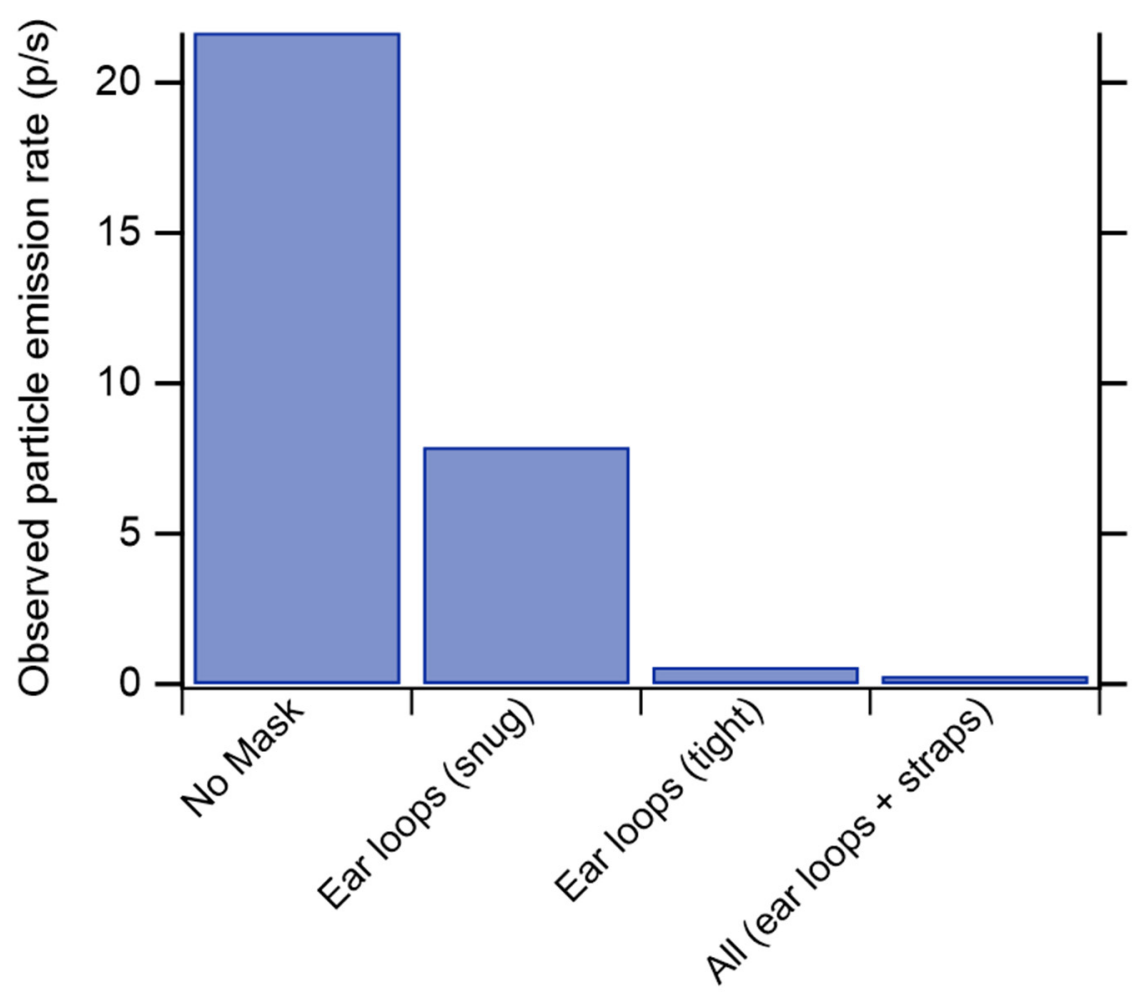

585 Figure S8. Observed particle emission rates $(\mathrm{p} / \mathrm{s})$ without a mask or for sampling from the top 586 quadrant with the singing mask worn with snug ear loops only, tight ear loops-only, or tight ear 587 loops plus the head straps. 


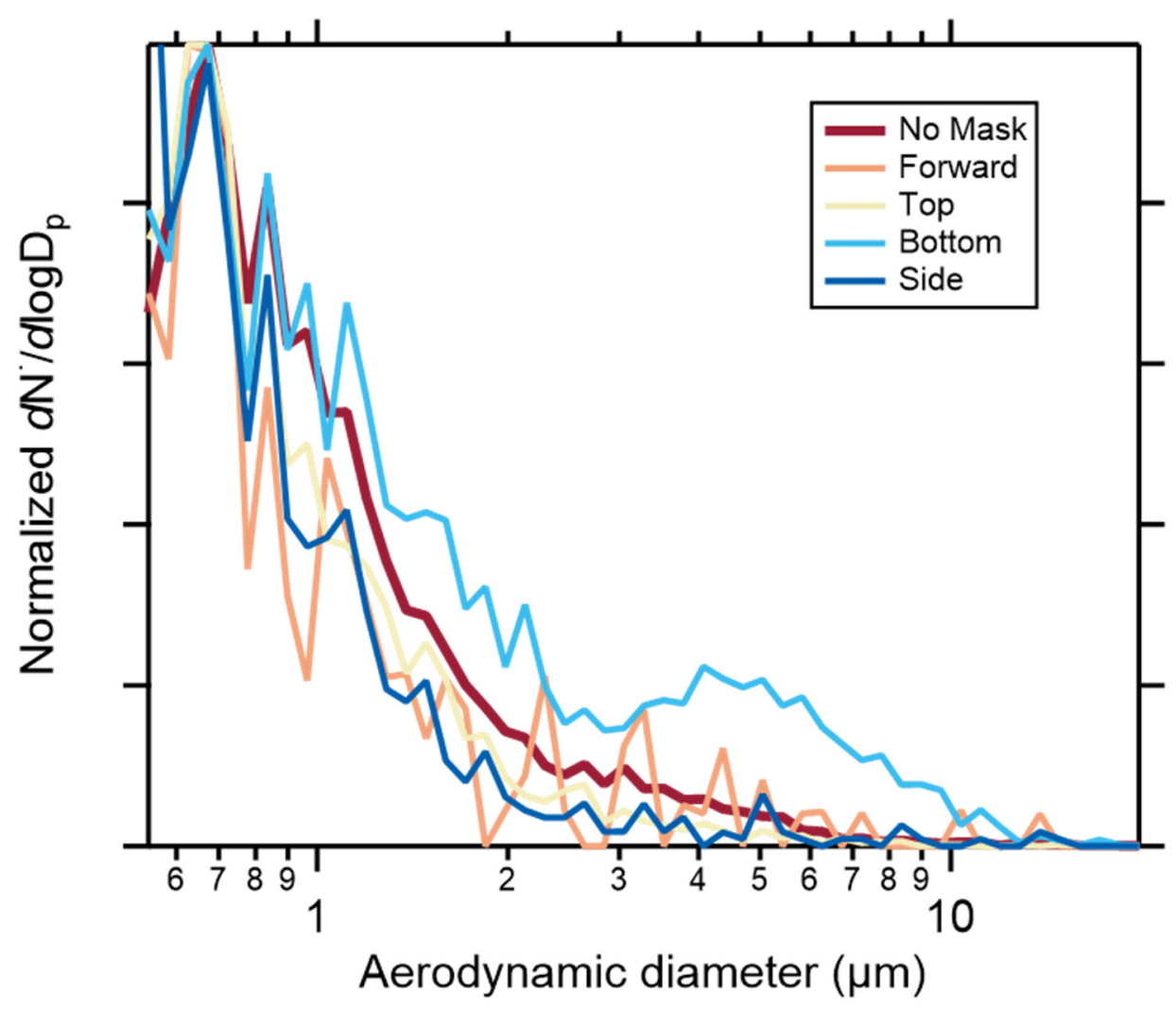

Figure S9. Normalized average particle emission rate size distributions for sampling with no mask 593 or with the singing mask from different quadrants. Note the enhanced contribution of large 594 particles (3-9 microns) when sampling from the bottom quadrant, indicative of fiber shedding. 


\section{Figures}
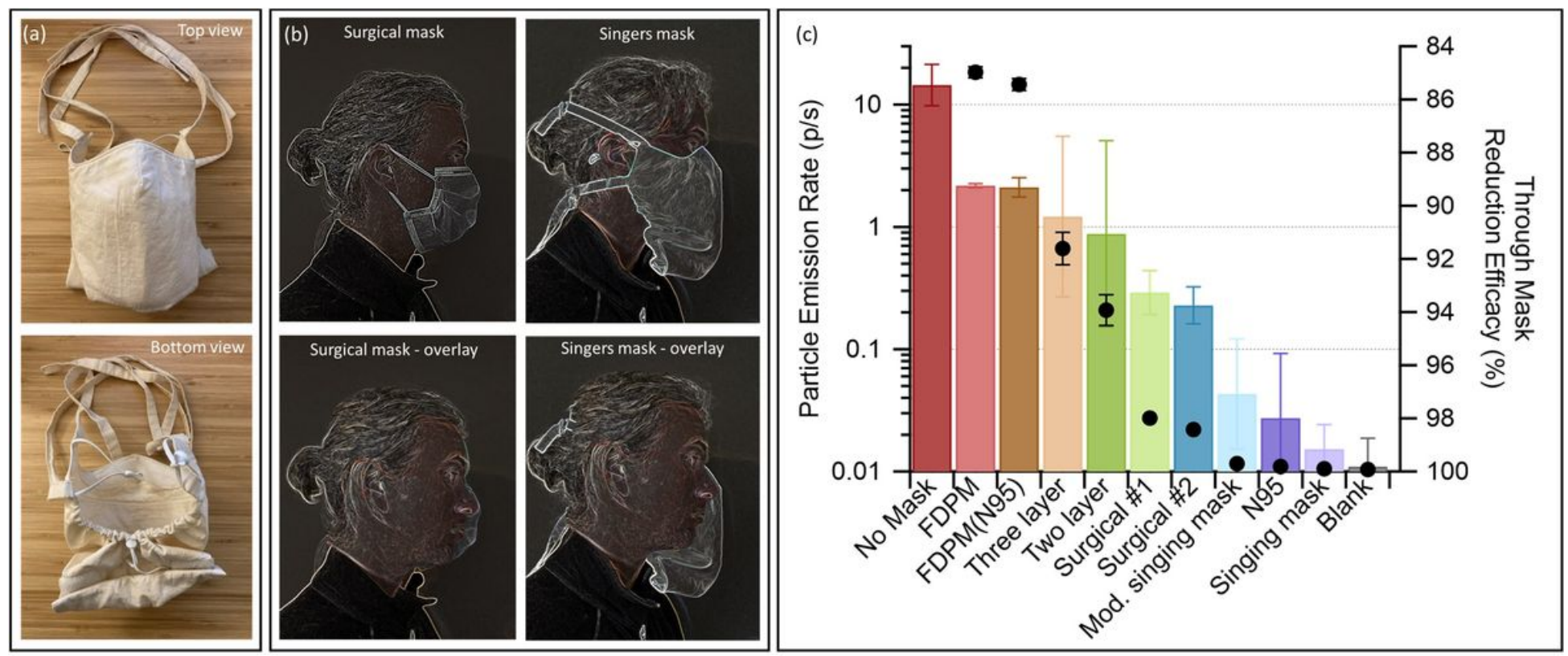

Figure 1

(a) Top and bottom view of the singing mask. (b) (top row) Images of one participant wearing a surgical mask and wearing the singing mask. (bottom row) Overlaid images of the participant with the surgical mask and with a singing mask to illustrate the increased mask-face material separation in the singing mask. The images in (b) were modified from the originals using the "glow edges" artistic effect in Microsoft PowerPoint to accentuate the mask edges and to overlay an image of the participant with no mask. Informed consent was obtained. (c) Observed particle emission rate for one participant singing Ode to Joy with no mask, or a homemade cloth two-layer or three-layer pleated mask made of the same material as the singing mask, a modified singing mask having no bottom opening, the standard singing mask (purple), a commercial cloth mask either without (FDPM) or with (FDPM(N95)) an N95 insert, either of two different surgical masks, or an N95 respirator (3M), as measured in the forward (through mask) position. Note that jaw movement was substantially restricted with the non-singing masks, especially the N95 respirator. Three repeats were performed and error bars are 10 geometric standard deviations. The right axis gives the associated reduction efficiency for the forward direction (black points). 


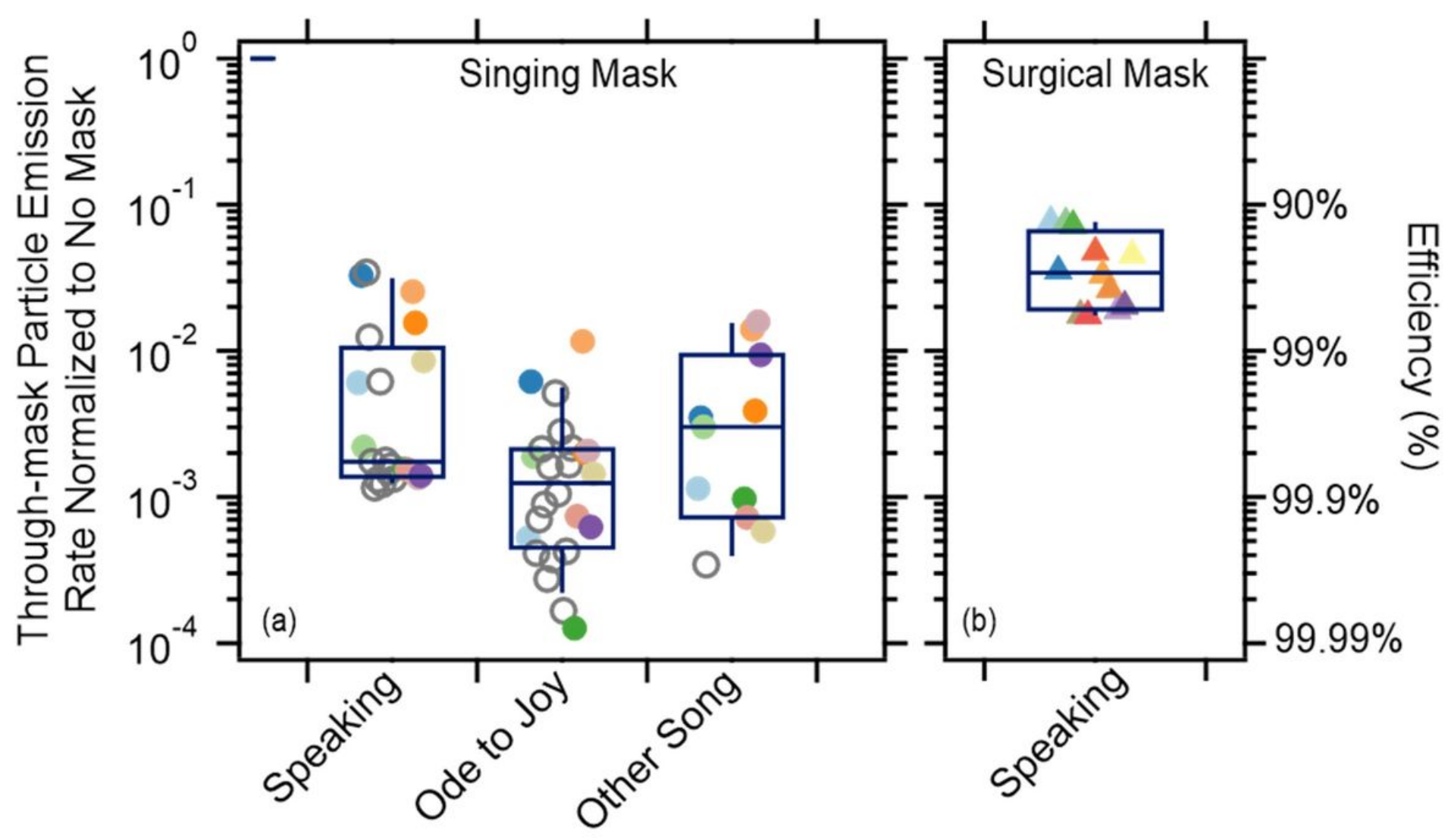

Figure 2

(a) The through-mask (forward) particle emission rates normalized to no mask (left axis) or the corresponding reduction efficiency (right axis) observed for participants while wearing the singing mask for speaking or singing either the Ode to Joy or a song chosen by the 501 participant. Closed colored circles in (a) indicate unique individuals while open gray circles 502 indicate repeats by one individual. (b) Normalized particle emission rates for participants 503 speaking while wearing a surgical mask (data from Cappa et al. (11)). Note that the participants 504 in (a) differ from those in (b). Box and whisker plots show the median, 25th/75th percentile, and 505 10th/90th percentile. 


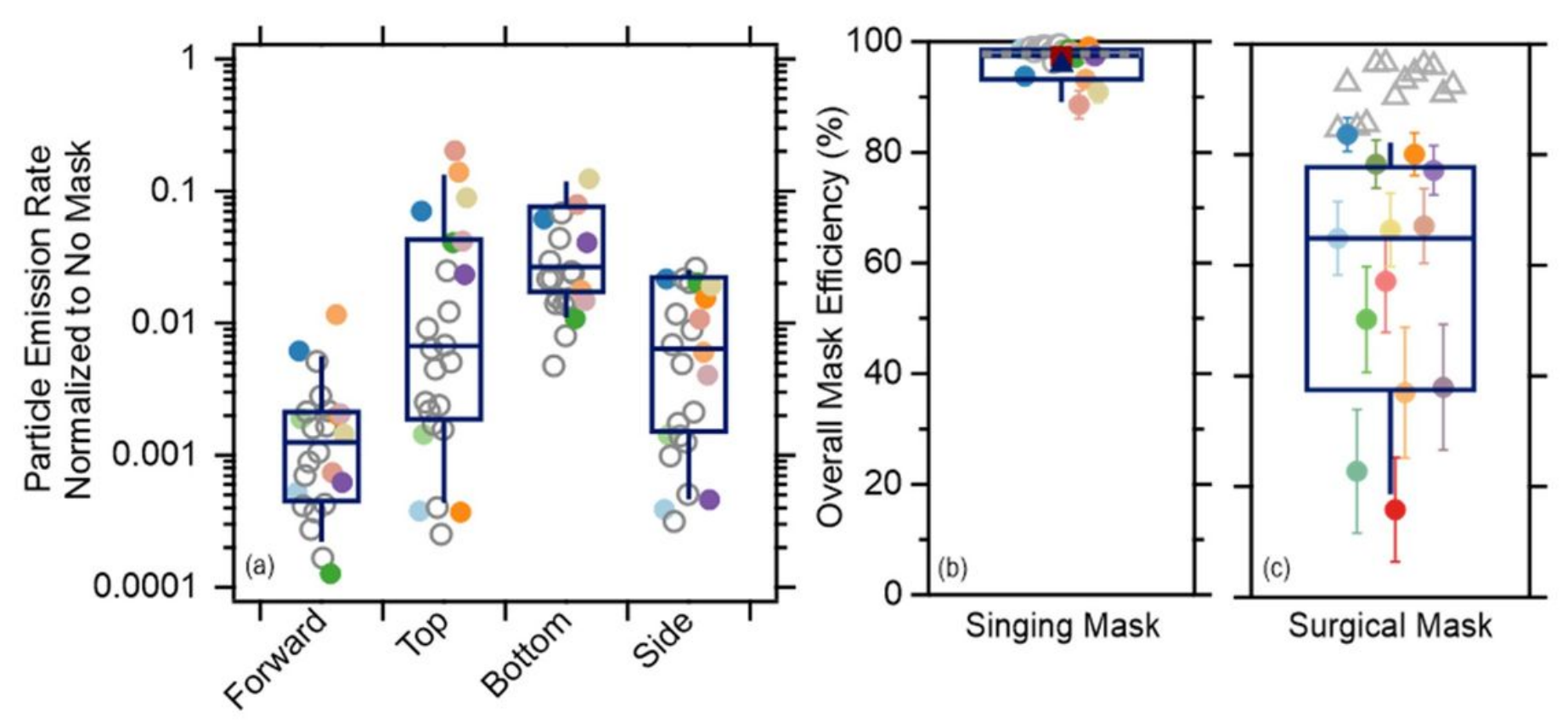

Figure 3

(a) Particle emission rates normalized to no mask wearing for singing Ode to Joy for the different orientations while wearing the singing mask. (b) The overall mask efficiency while singing Ode to Joy with the singing mask, accounting for leakage flows, colored by participant (circles). Uncertainty bars on individual points indicate 10 determined from Monte Carlo simulations. The red square is the average across all measurements and the blue triangle the average after combining replicate measurements from one participant. (c) Overall efficiency for speaking with a surgical mask including leakage flows (solid points) or assuming all flow passes through the mask, i.e. zero leakage flows and perfect sealing (open points). Closed circles in (b) and (c) indicate unique individuals while open circles indicate repeats by one individual. Note that the participants in $(a, b)$ differ from those in (c). Box and whisker plots show the median, 25th/75th percentile, and 10th/90th percentile. 


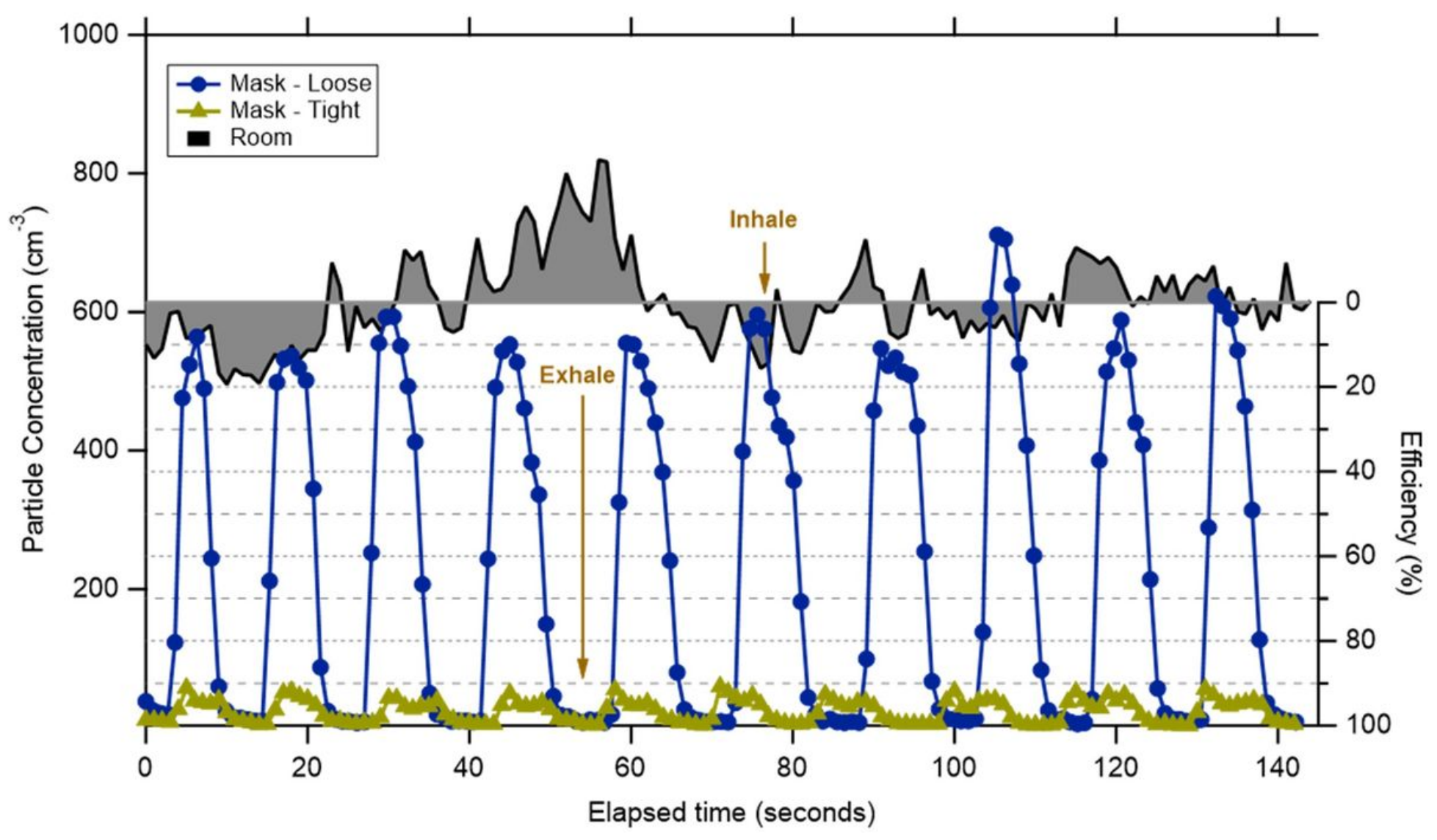

Figure 4

Time-series of particle concentrations measured for room air (solid black line), sampled inside the singing mask worn intentionally loose around the neck (blue circles), and when sampled inside the mask when worn tightly as designed (gold triangles). The right axis shows the approximate mask efficiency based on comparison with the mean room air concentration. 